\title{
Hepatoprotective Effect of Quercetin on Endoplasmic Reticulum Stress and Inflammation after Intense Exercise in Mice through Phosphoinositide 3-Kinase and Nuclear Factor-Kappa B
}

\author{
Yuhan Tang, ${ }^{1}$ Juan Li, ${ }^{1}$ Chao Gao, ${ }^{1,2}$ Yanyan $\mathrm{Xu},{ }^{1}$ Yanyan Li, ${ }^{1}$ \\ Xiao Yu, ${ }^{1}$ Jing Wang, ${ }^{1}$ Liegang Liu, ${ }^{1}$ and Ping Yao ${ }^{1}$ \\ ${ }^{1}$ Department of Nutrition and Food Hygiene, Hubei Key Laboratory of Food Nutrition and Safety, Ministry of \\ Education Key Laboratory of Environment and Health and MOE Key Lab of Environment and Health, Key Laboratory of \\ Environment and Health (Wuhan), Ministry of Environmental Protection, and State Key Laboratory of \\ Environment Health (Incubation), School of Public Health, Tongji Medical College, Huazhong University of Science and Technology, \\ Wuhan 430030, China \\ ${ }^{2}$ National Institute for Nutrition and Food Safety, Chinese Center for Disease Control and Prevention, Beijing 102206, China
}

Correspondence should be addressed to Ping Yao; yaoping@mails.tjmu.edu.cn

Received 13 March 2016; Accepted 19 June 2016

Academic Editor: Ryuichi Morishita

Copyright (c) 2016 Yuhan Tang et al. This is an open access article distributed under the Creative Commons Attribution License, which permits unrestricted use, distribution, and reproduction in any medium, provided the original work is properly cited.

\begin{abstract}
The mechanisms underlying intense exercise-induced liver damage and its potential treatments remain unclear. We explored the hepatoprotection and mechanisms of quercetin, a naturally occurring flavonoid, in strenuous exercise-derived endoplasmic reticulum stress (ERS) and inflammation. Intense exercise $\left(28 \mathrm{~m} / \mathrm{min}\right.$ at a $5^{\circ}$ slope for $\left.90 \mathrm{~min}\right)$ resulted in the leakage of aminotransferases in the BALB/C mice. The hepatic ultrastructural malformations and oxidative stress levels were attenuated by quercetin $(100 \mathrm{mg} / \mathrm{kg} \cdot \mathrm{bw})$. Intense exercise and thapsigargin- (Tg-) induced ERS (glucose-regulated protein 78, GRP78) and inflammatory cytokines levels (IL-6 and TNF- $\alpha$ ) were decreased with quercetin. Furthermore, quercetin resulted in phosphoinositide 3-kinase (PI3K) induction, $\mathrm{Ca}^{2+}$ restoration, and blockade of the activities of Jun $\mathrm{N}$-terminal kinase (JNK), activating transcription factor 6 (ATF6) and especially NF- $\kappa \mathrm{B}$ (p65 and p50 nuclear translocation). A PI3K inhibitor abrogated the protection of quercetin on ERS and inflammation of mouse hepatocytes. SP600125 (JNK inhibitor), AEBSF (ATF6 inhibitor), and especially PDTC (NF- $\kappa$ B inhibitor) enhanced the quercetin-induced protection against Tg stimulation. Collectively, intense exercise-induced ERS and inflammation were attenuated by quercetin. PI3K/Akt activation and JNK, ATF6, and especially NF- $\kappa$ B suppression were involved in the protection. Our results highlight a novel preventive strategy for treating ERS and inflammationmediated liver damage induced by intense exercise using natural phytochemicals.
\end{abstract}

\section{Introduction}

Moderate exercise provides benefits to health, weight, and mood management. Accumulating evidence has demonstrated that regular physical activity enhances the antioxidant and anti-inflammatory properties and functional capacities of various tissues and organs. These factors minimize or prevent chronic problems, including the common nonalcoholic fatty liver disease [1]. Paradoxically, strenuous, prolonged, or intense exercises have been shown to be strong stressors with negative consequences, indicating a " $J$ - or U-shaped association" between health and exercise; thus, there remains an incomplete understanding of the dose-response relationship between exercise and health [2]. Intolerable overexercise has been frequently associated with the occurrence of overtraining syndrome, which manifests as fatigue, impaired immune functions, repetitive tissue trauma, inflammation of muscles and viscera, and chronic health issues [3]. Injury and dysfunction of the liver, a vital organ responsible for the metabolism of nutrients and biochemical, inflammatory mediators and 
the detoxification of toxic substances, were first reported by Fojt et al. [4]. Subsequently, inflammation, oxidative stress, and disturbed lipid metabolism in animals and human models following vigorous overexercise have been reported in other studies [5-7]. In contrast with the widely studied exercise-induced heart injury models [8], studies of liver damage have drawn little attention due to the lack of evident clinical symptoms in early stages. A lack of understanding of liver disorders may impede the management of athlete health. Additionally, to prevent progression to advanced and fatal liver damage and thus to develop early intervention strategies, it is imperative to explore the molecular mechanisms underlying liver damage especially on endoplasmic reticulum stress (ERS) and inflammation induced by overexercise.

Recently, ERS has attracted particular interest due to its role in inducing inflammatory responses under various pathological conditions. Of note, moderate treadmill running has been found to attenuate skeletal ERS and improve insulin sensitivity in rats with insulin resistance [9]. Furthermore, moderate exercise has been shown to repress neuronal ERS and inflammation in aged presenilin 2 mutant mice [10]. Deldicque et al. [11] have found that endurance training promotes the unfolded protein response but attenuates inflammation in the soleus and tibialis anterior muscles and in the livers and pancreases of rats fed high-fat diets, suggesting a positive protective adaptation against further cellular stress. However, few studies have addressed the role of ERS in exhaustive exercise-related disorders, especially liver damage. Kim and his colleagues have observed evident ERS, oxidative stress, and inflammation in biopsies of vastus lateralis muscles after $200 \mathrm{~km}$ runs [12], further implying a link between ERS and overexercise. However, nuclear factor-kappa B (NF- $\kappa \mathrm{B})$, Jun $\mathrm{N}$-terminal kinase (JNK), and activating transcription factor 6 (ATF6) are considered crucial signaling pathways for ERS-initiated inflammation $[9,13]$. Intense exercise has been reported to induce $\mathrm{NF}-\kappa \mathrm{B}$ phosphorylation in human skeletal muscle [14] and ATF6 expression upregulation in the mouse hypothalamus, hippocampus, and cortex [15]. However, it has also been shown to inhibit JNK phosphorylation in the rat adrenal medulla [16]. The correlation between ERS and inflammatory damage and potential signaling pathways involved during vigorous exercise remain unclear.

Quercetin is the most common and widely distributed flavonoid in the plant kingdom, and it is especially abundant in apples (ranging from 2.1 to $7.2 \mathrm{mg} / 100 \mathrm{~g}$ ) and onions (ranging from 28.4 to $48.6 \mathrm{mg} / 100 \mathrm{~g}$ ). According to epidemical survey, the daily media intake of quercetin with a typical Western diet and in China was estimated to $10 \mathrm{mg}$ and $5.96 \mathrm{mg} / \mathrm{day}$, respectively [17]. In order to maximally exert its biological effect, quercetin should be assumed as purified dietary supplement but not natural food source. Quercetin has been found to have applicative uses in the military, athletic, and elderly populations and has also been considered in the preventive and therapeutic treatments of conditions and diseases related to oxidative and inflammatory damage. A systematic review [18] that pooled 11 studies totaling 254 human subjects has confirmed that quercetin provides significant benefits to human endurance exercise capacity and performance, despite variations in experimental designs [19]. Emerging evidence from our and other groups has shown that quercetin exerts hepatoprotective effects due to its antioxidant capacity, antiinflammatory activity, and gene regulatory properties [19-21]. Furthermore, it has been suggested to alleviate inflammation by suppressing NF- $\kappa \mathrm{B}$ and, to some extent, JNK pathways [22, 23]. Moreover, Liu et al. [21] have found that quercetin prevents $\mathrm{Pb}$-induced ERS at least in part due to its modulation of phosphoinositide 3-kinase (PI3K)/protein kinase B (Akt) and inositol-requiring enzyme 1 (IRE1)/JNK signaling pathways. However, the mechanistic role of the PI3K/Akt signaling pathway in the quercetin-mediated protection against intense exercise-induced ERS remains unclear. Furthermore, the mechanism responsible for transducing ERS signals into inflammatory injury has yet to be identified.

We designed the current study to determine the potential hepatoprotective effect of quercetin against intense exerciseinduced hepatic ERS and inflammation damage. Furthermore, we characterized the underlying mechanisms involved in the regulation of the PI3K/Akt and ERS transducers (i.e., $\mathrm{NF}-\kappa \mathrm{B}$, JNK, and ATF6) during overexercise and in response to intervention by phytochemicals.

\section{Materials and Methods}

2.1. Chemicals and Materials. Quercetin ( $\geq 98 \%$, HPLC), IV collagenase, thapsigargin ( $\mathrm{Tg}$ ), pyrrolidine dithiocarbamate (PDTC), SP600125, 4-(2-aminoethyl)benzenesulfonyl fluoride hydrochloride (AEBSF), and LY294002 were obtained from Sigma-Aldrich (Saint Louis, Missouri, USA). Assay kits for aspartate/alanine transaminase (AST/ALT) were purchased from Mindray (Shenzhen, China). Anti-glucose-regulated protein 78 (GRP78) rat monoclonal antibody was obtained from Santa Cruz Biotechnology (Santa Cruz, California, USA). Rabbit monoclonal IL, interleukin-6 (IL-6), PI3K (p85), Akt and phospho-Akt (Ser473) antibodies, anti-JNK rabbit monoclonal antibody, anti-p-JNK mouse monoclonal antibody, anti-TATA binding protein (TBP) rabbit polyclonal antibody, horseradish peroxidase- (HRP-) conjugated goat anti-rat IgG (secondary antibody), anti-HRP-conjugated goat anti-rabbit IgG, and anti-HRP-conjugated horse anti-mouse IgG were acquired from Cell Signaling Technology (Danfoss, Massachusetts, USA). Anti-glyceraldehyde-3-phosphate dehydrogenase (GAPDH) rabbit polyclonal antibody was obtained from Epitomics (Burlingame, California, USA). Anti-IRE1 $\alpha$ rabbit polyclonal antibody, anti-p-IRE1 $\alpha$ rabbit polyclonal antibody, anti-p-protein kinase RNA-activatedlike ER kinase (PERK) rabbit monoclonal antibody, and antitumor necrosis factor (TNF- $\alpha$ ) mouse monoclonal antibody were purchased from Abcam (Cambridge, British). Anti-p65 rabbit monoclonal antibody and anti-p50 rabbit polyclonal antibody were obtained from Cell Signaling and Protein Tech (Chicago, Illinois, USA), respectively. Western blot detection reagents (ECL) and reblot buffer were provided by Chemicon (Temecula, CA, USA). IL-6 and TNF- $\alpha$ ELISA kits are acquired from R\&D Systems and Genzyme, respectively. Microsomal $\mathrm{Ca}^{2+}$ concentration determination and nuclear protein extraction kits were purchased from Genemed and Thermo, respectively. An ATF6 activity determination ELISA kit was obtained from Uscn Life Science. Other chemicals and 
organic solvents were of analytical grade and were purchased from local reagent retailers.

2.2. Animal Treatment and Exercise Protocol. Forty male adult BALB/C mice, obtained from Sino-British Sippr/BK (Shanghai, China) and with body weights of $18-20 \mathrm{~g}$, were randomly divided into the following four groups containing ten animals each: (1) rested control (Ct); (2) intense exercise (Ex); (3) intense exercise plus quercetin supplementation (Ex $+\mathrm{Qu})$; and (4) rested plus dietary quercetin supplementation $(\mathrm{Qu})$ groups. Based on our preliminary test, quercetin was administered every day throughout the experiment (5 weeks) by gavage at a dose of $100 \mathrm{mg} / \mathrm{kg} \cdot \mathrm{bw}(10 \mathrm{~mL} / \mathrm{kg} \cdot \mathrm{bw})$, dissolved in physiological saline. The animals were subjected to an intense exercise program on the fourth week according to Marra et al. with some modifications [24]. The mice were forced to run on a motor-driven treadmill (Anhui, China) once a day for 7 successive days followed by a two-day rest period. They were acclimated to the treadmill exercise before training for two consecutive days. The exercise intensity (running speed) was set to a speed of $10 \mathrm{~m} / \mathrm{min}$ for $10 \mathrm{~min} /$ day for the two acclimation days, after which time the animals were subjected to daily sessions of intense exercise for $28 \mathrm{~m} / \mathrm{min}$ for $90 \mathrm{~min} /$ day following a $10 \mathrm{~min}$ warm-up period.

The animals were cared for according to the Guiding Principles of the Care and Use of Laboratory Animals published by the US National Institutes of Health. Animal experiments described in this study were approved by the Institutional Animal Care and Use Committee at Tongji Medical College, HUST (IACUC number: S407). The mice were housed in a temperature-regulated room $\left(20-25^{\circ} \mathrm{C}\right)$ at a relative humidity level (60-75\%) with a 12-hour light/dark cycle and provided with food and water ad libitum. The health statuses of the mice were monitored throughout the experiment by measuring the body weights on a weekly basis, in addition to monitoring food consumption, physical activity, and other measurable signs (general appearance, hair, eyes, nose, feces, urine, etc.) daily. The mice were immediately euthanized using a sodium pentobarbital solution ( $40 \mathrm{mg} / \mathrm{kg} \cdot \mathrm{bw}, 1 \%$ sodium pentobarbital dissolved in physiological saline, $4 \mathrm{~mL} / 1 \mathrm{~kg} \cdot \mathrm{bw}$, ip) after the final exercise session. Serum samples were prepared from blood by centrifugation at $3500 \mathrm{~g}$ for $10 \mathrm{~min}$ at $4^{\circ} \mathrm{C}$ (Eppendorf 5810R, Hamburg, Germany). Fresh liver samples were fixed for histopathological examination, and flash-frozen samples were used for Western blot and enzymatic analyses.

\subsection{Determination of Serum Aminotransferase and Cytokine} Levels and Hepatic Oxidative Stress Status. The serum levels of aminotransferases (AST and ALT) and inflammatory markers (TNF- $\alpha$ and IL-6) were measured by enzymatic kinetic methods and enzyme-linked immunosorbent assays according to the manufacturer's instructions with the relevant kits, respectively.

Malondialdehyde (MDA), ROS, reduced glutathione (GSH), and superoxide dismutase (SOD) activities were determined as previously described $[25,26]$. Liver homogenate with isotonic saline (10\%) was centrifuged at $3500 \mathrm{~g}$ for $10 \mathrm{~min}$ $\left(4^{\circ} \mathrm{C}\right)$ to obtain the supernatant. The hepatic ROS assay was based on the oxidation of dihydroethidium (DHE), yielding a red fluorescent product, which was observed with a Nikon 2000 S fluorescence microscope (Melville, NY) and analyzed by Image-Pro Plus software. The specific activities of the various enzymes and the MDA concentration in the liver were normalized to the total protein concentration and expressed as $\mathrm{nmol} / \mathrm{mg}$ protein.

2.4. Liver Ultrastructural Examination. Exercise-induced ultrastructural changes were observed by conventional methods. Briefly, fresh liver fragments (1 mm cubes) were carefully collected, rapidly fixed in $2.5 \%$ glutaraldehyde (in phosphate buffer, $\mathrm{pH}$ 7.4), and then postfixed in $1 \%$ osmium tetroxide. Subsequently, the samples were dehydrated by graded alcohol and embedded in Epon 812 resin. Ultrathin sections were prepared using an Ultramicrotome (Leica UCT, Germany). The sections were stained with uranyl acetate and lead citrate and were subsequently visualized by transmission electron microscopy (FEI Tecnai G2 12, Netherlands). Digital images were captured with an integrated CCD camera.

2.5. Microsomal Extraction and $\mathrm{Ca}^{2+}$ Concentration Determination. Microsomal samples were extracted from the liver homogenate $(0.1 \mathrm{M}$ potassium phosphate buffer) by differential ultraspeed centrifugation $(3500 \mathrm{~g}$ for $10 \mathrm{~min}, 10,800 \mathrm{~g}$ for $15 \mathrm{~min}$, and 105,000 g for $60 \mathrm{~min}$; Beckman, Optima L-90K, USA) at $4^{\circ} \mathrm{C}$, according to previously reported methods [25]. $\mathrm{Ca}^{2+}$ concentrations were measured by fluorescence spectrophotometry using Fluo-4-AM.

2.6. Isolation, Culture, and Treatment of Mouse Hepatocytes. Mouse hepatocytes were isolated by a two-step collagenase perfusion technique from the abdominal aorta. The perfusions were purified via a Percoll gradient, as described by Cao et al. [27]. Freshly harvested hepatocytes were cultured on rat-tail collagen-coated plates and maintained in high glucose DMEM supplemented with glutamine, $10 \%$ fetal bovine serum, $32 \mathrm{IE} / \mathrm{L}$ insulin, $15 \mathrm{mM}$ HEPES, $0.1 \mu \mathrm{M}$ hydrocortisone, $100 \mathrm{U} / \mathrm{mL}$ penicillin, and $100 \mathrm{U} / \mathrm{mL}$ streptomycin. The next day, the media were exchanged and supplemented with Tg $(1 \mu \mathrm{M})$, PDTC $(300 \mu \mathrm{M})$, SP600125 $(20 \mu \mathrm{M})$, AEBSF $(300 \mu \mathrm{M})$, LY294002 $(30 \mathrm{nM})$, and quercetin $(100 \mu \mathrm{M})$ for 24 hours. Then, the cells and supernatants were collected for bioassays performed according to the corresponding experimental protocols. Cell viability was $80-95 \%$ (as determined by the trypan blue exclusion test) under these conditions.

2.7. Immunohistochemistry. Liver tissue samples fixed in $4 \%$ paraformaldehyde/PBS were embedded in paraffin and cut into $5 \mu \mathrm{m}$ thick sections. The tissue sections used to determine GRP78 expression were incubated with the appropriate primary antibody (diluted $1: 100$ ) overnight at $4^{\circ} \mathrm{C}$. The immunostaining was visualized using $3,3^{\prime}$-diaminobenzidine following a 1-hour reaction with an HRP-conjugated-IgG antibody (diluted 1:200).

2.8. Real-Time Quantitative PCR Analysis. Total RNA was extracted from mouse liver tissues using TRIzol $^{\circledR}$ reagent 
(Invitrogen, Carlsbad, CA) according to the manufacturer's instructions. The messenger RNA (mRNA) levels of the target genes were quantified by qRT-PCR using a SYBR greenbased kit (TaKaRa BIO Inc., Dalian) with specific primers and an RT-PCR machine (7900HT, Applied Biosystems, Forster, CA). The mRNA level of $\beta$-actin served as an endogenous control and the results were calculated by the comparative $2^{-\Delta \Delta \mathrm{Ct}}$ method. The forward and reverse primers for IL-6 (NM_031168.1) were GGG ACT GAT GCT GGT GAC AA and ACA GGT CTG TTG GGA GTG GT, respectively. In addition, the primers for TNF- $\alpha$ (NM_013693.3) were ATG GCC TCC CTC TCA GT and TTT GCT ACG TGG GCT AC, and those for $\beta$-actin (NM_007393.3) were TTC GTT GCC GGT CCA CAC CC and GCT TTG CAC ATG CCG GAG CC.

2.9. Western Blot Analysis. Mouse hepatocytes and liver tissues were lysed in RIPA Lysis Buffer (1\% Triton X-100, $1 \%$ deoxycholate, and $0.1 \%$ SDS) or using a cell plasma and nuclear protein extraction kit according to the manufacturer's instructions. Tissue or cellular lysates with equal amounts of proteins mixed $(3: 1)$ with loading buffer were subjected to electrophoresis (Bio-Rad, USA) in acrylamide-SDS gels and subsequent electroblotting to polyvinylidene fluoride membranes (Millipore, Massachusetts, USA). The target proteins were probed with specific primary antibodies. Then, the blot membranes were incubated with species-specific HRPconjugated secondary antibodies. The chemiluminescence intensities of the bands were subsequently detected using an ECL Plus Kit with a Western Blotting Detection System (Amersham Biosciences, Little Chalfont, UK). The optical densities of the bands were quantified by Gel Pro 3.0 software (Biometra, Goettingen, Germany). To eliminate background noise, the data were standardized to GAPDH as optical density values $\left(\mathrm{OD} / \mathrm{mm}^{2}\right)$.

2.10. Statistical Analysis. The data were expressed as the mean \pm standard deviation (SD) and were analyzed using SPSS 12.0 software package by one-way analysis of variance (ANOVA). A probability value of 0.05 (two-tailed) was considered significant.

\section{Results}

All of the mice survived for the entire experimental period until they were sacrificed. There were no significant differences in the initial body weights among the 4 experimental groups. However, the weight loss of the overexercisechallenged mice was greater than that of nonoverexercised mice. Additionally, quercetin supplementation did not have an effect on weight change in the overexercise-exposed mice (Supplementary Figure 1; see Supplementary Material available online at http://dx.doi.org/10.1155/2016/8696587).

3.1. Quercetin Decreased Aminotransferase Release and Oxidative Stress and Alleviated Hepatic Ultrastructural Abnormalities in Overexercised Mice. Intense exercise resulted in 1.6fold and 1.4-fold increases in the serum ALT and AST levels, respectively, in the mice compared with those in the normal controls. The amounts of serum ALT and AST released from the intense exercise-exposed mice were decreased by $35 \%$ and $25 \%$, respectively, as a result of daily quercetin pretreatment (Figures 1(a) and 1(b)). The MDA and ROS levels were increased in the intense exercise-challenged mice compared with the control mice (Figures 1(c) and 1(d)). Notably, tripeptide GSH was significantly consumed and depleted during intense exercise (Figure 1(e)), whereas a substantial increase in SOD activity was observed (Figure 1(f)). The pretreatment with quercetin reduced the accumulation of MDA and ROS and recovered the generation of GSH disturbed by rigorous exercise. Interestingly, quercetin supplementation further enhanced the activity of SOD induced by intense exercise.

To further evaluate the hepatoprotective effects of quercetin, ultrastructural examination was conducted by transmission electron microscopy (Figure $1(\mathrm{~g})$ ). Well-arranged rough endoplasmic reticula (RERs) with abundant attached ribosomes were observed in hepatocytes from the normal mice treated with or without quercetin. These structures were accompanied by many well-developed mitochondria with integral membranes and cristae. The nuclei of these hepatocytes were round with clear nuclear membranes, perinuclear cisterna, and nucleopores. Additionally, glycogenosomes were uniformly distributed. Intense exercise resulted in various degenerative changes. Fewer ribosomes were observed attached to swollen RERs (Figure $1(\mathrm{~g}) \mathrm{R}$ ); mitochondria appeared dilated or malformed (with missing cristae; Figure 1(g) M); and the number of glycogenosomes was reduced. These intense exercise-induced ultrastructural abnormalities were somewhat reversed by quercetin; but the mitochondria remained swollen. The treatment of normal, nonoverexercised mice with quercetin had no effects on the serum aminotransferase levels, hepatic oxidative stress, or ultrastructural changes.

\subsection{Quercetin Attenuated Intense Exercise-Induced Inflamma-} tory Damage and ERS of Mouse Liver. Inflammatory stress was first evaluated in the present study. Markedly augmented hepatic IL- 6 and TNF- $\alpha$ mRNA levels (Figures 2(a) and 2(b)) and increased serum inflammatory cytokine levels (Figures 2(c) and 2(d)) were observed in the intense exercisechallenged mice compared with the normal control mice. Subsequently, hepatic ERS, an initial cause of inflammation, was investigated. Hepatic GRP78, a classic ERS marker [28], was measured by Western blot and immunohistochemistry analyses. In correlation with inflammation, GRP78 protein expression was significantly upregulated following intense exercise (Figures 3(a), 3(b), and 3(c)), indicating that ERS potentially initiates inflammation in intense exercise-elicited liver injury. Furthermore, decreased expression of p-IRE$1 \alpha$ and increased level of p-PERK protein (Figures 3(b) and $3(\mathrm{~d})$ ), which are responsible for ERS-associated signaling [27], were observed in the intense exercise-exposed mice compared with control mice. The treatment of these intense exercise-exposed mice with quercetin had potent hepatoprotective effects via the inhibition of GRP78 and p-PERK, which increased the expression of $\mathrm{p}$-IRE-1 $\alpha$ and reduced the levels of inflammatory factors. These observations further supported 


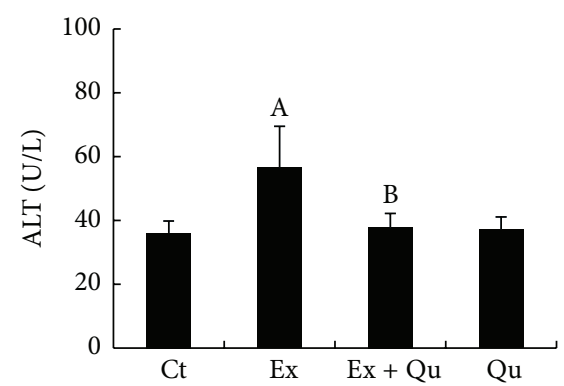

(a)

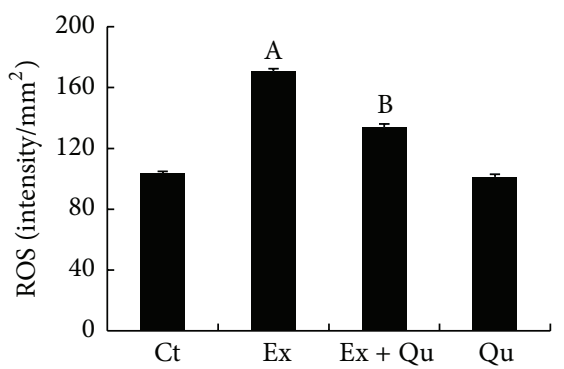

(d)

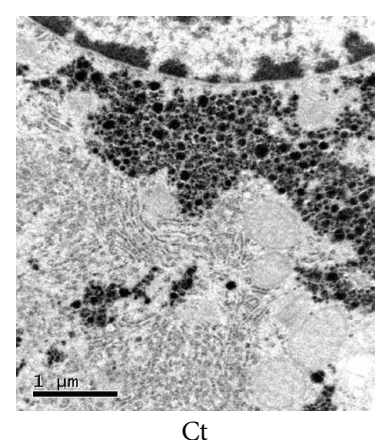

Ct

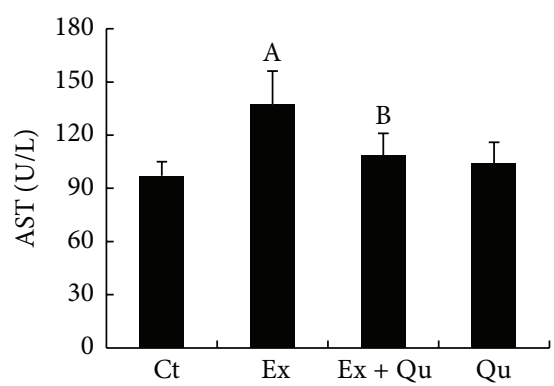

(b)

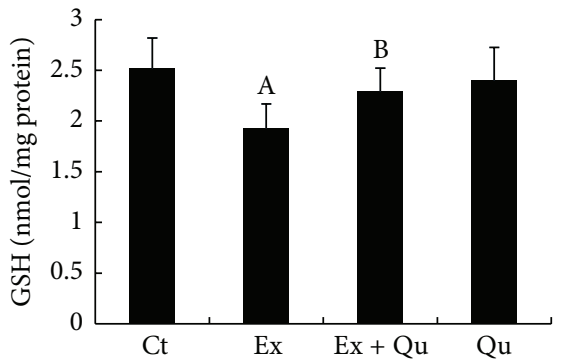

(e)

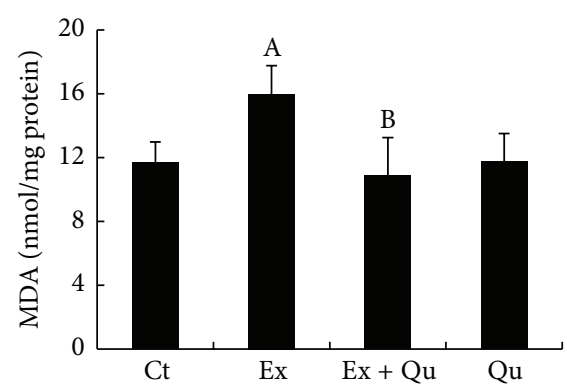

(c)

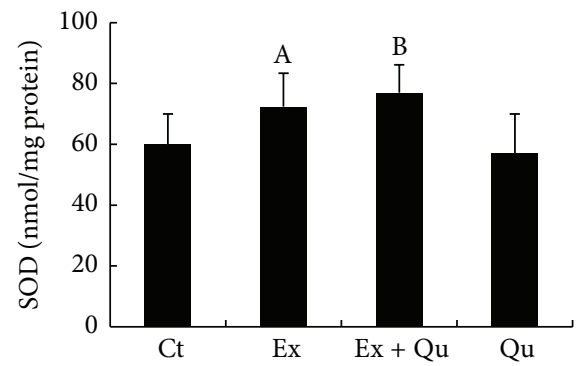

(f)

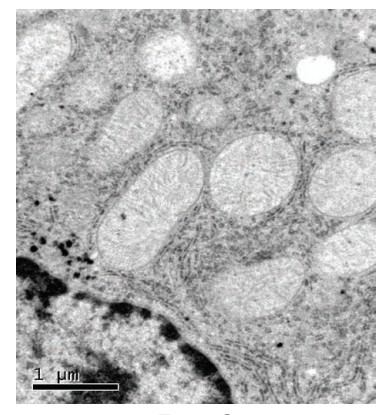

$\mathrm{Ex}+\mathrm{Qu}$

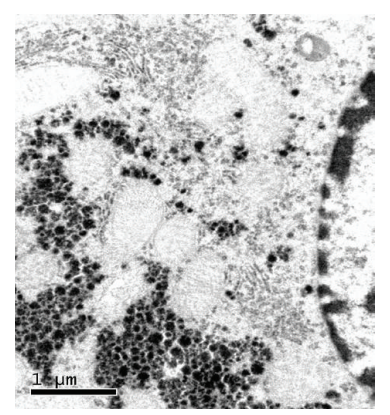

$\mathrm{Qu}$

(g)

FIGURE 1: Effects of intense exercise exposure and quercetin pretreatment on serum aminotransferase levels, oxidative stress, and liver ultrastructural changes. Serum ALT (a) and AST (b) levels were measured using standard commercial assay kits. Quantification of MDA (c), ROS (d), GSH (e), and SOD (f) levels in liver tissues; the bars represent the mean \pm SD $(N=3$ for ROS determination, $N=10$ for others). (g) Hepatic ultrastructures were observed by transmission electron microscopy. Significant differences $(P<0.05)$ are indicated by the different letters. A: versus rested control (Ct); B: versus intense exercise (Ex); Ex + Qu: intense exercise plus quercetin (100 mg/kg.bw); Qu: quercetin.

the hypothesized link between ERS and inflammation. However, quercetin by itself had no effects on ERS or inflammatory damage compared with the normal, nontreated mice.

\subsection{PI3K/Akt Pathway Potentially Mediated the Protective} Effects of Quercetin against ERS. The role of the PI3K/Akt pathway, which has been extensively associated with ERS [29] but has not been considered in overexercise models, in quercetin-induced hepatoprotection against ERS elicited by intense exercise remains unknown. As depicted in Figures 4(a), 4(b), and 4(c), the hepatic PI3K and p-Akt expression levels were significantly decreased with intense exercise and were accompanied by a decreased $\mathrm{Ca}^{2+}$ concentration in the ER compared with normal control mice (Figure 4(d)). The latter effect has been proposed to sequester GRP78 away from ERS transducers and accordingly promote ERS [30]. In contrast, quercetin supplementation effectively abolished the effects of intense exercise on PI3K/Akt expression and the corresponding $\mathrm{Ca}^{2+}$ dynamics.

To further elucidate the roles of the PI3K/Akt pathway in quercetin-mediated protection against ERS and inflammation-related damage, mouse primary hepatocytes were treated with quercetin, $\mathrm{Tg}$ (an activator of ERS via $\mathrm{Ca}^{2+}$ dynamic dysregulation), and LY294002 (an irreversible PI3Kspecific inhibitor) following determination of the Tg concentration that stimulated ERS (in a dose-dependent manner, i.e., $0,0.1,0.5$, and $1.0 \mu \mathrm{M}$; data not shown). Similar results for GRP78 expression and inflammatory stress induced by Tg treatment were observed in the intense exercise-exposed mice. As expected, the quercetin intervention effectively reduced ERS and inflammation compared with the Tg-stimulated hepatocytes. Nevertheless, the protective effects of 


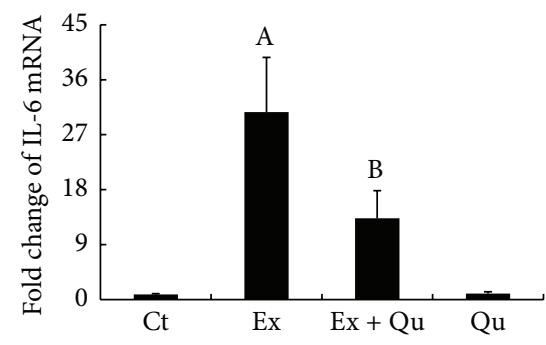

(a)

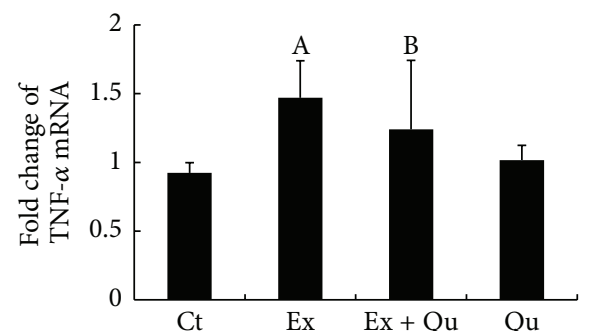

(b)

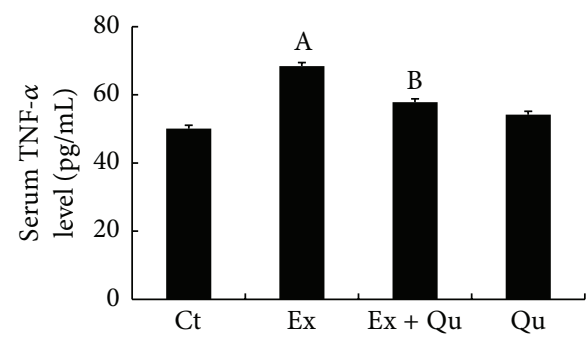

(d)

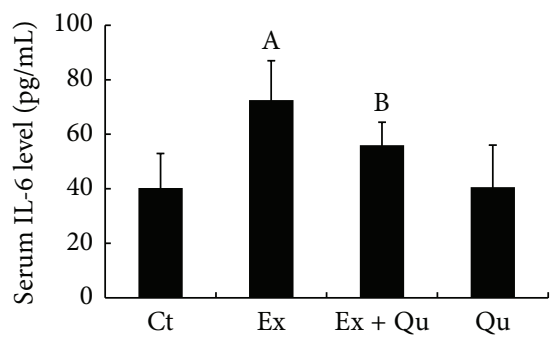

(c)

FIGURE 2: Influence of quercetin on inflammatory cytokines against intense exercise exposure in mouse liver. IL-6 (a) and TNF- $\alpha$ (b) mRNA expression levels; and IL-6 (c) and TNF- $\alpha$ (d) serum levels. The data are presented as the mean \pm SD $(N=8$ for ELISA, $N=6$ for mRNA). Significant differences $(P<0.05)$ are indicated by different letters. A: versus Ct; B: versus Ex.

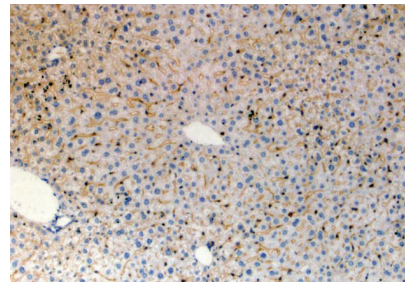

$\mathrm{Ct}$

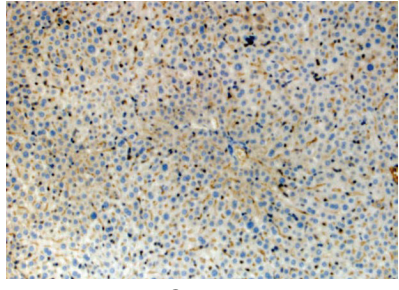

$\mathrm{Qu}$

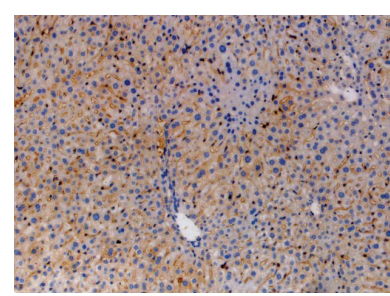

Ex

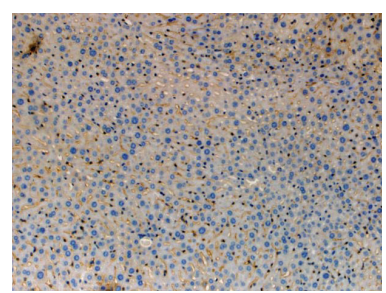

$\mathrm{Ex}+\mathrm{Qu}$

(a)

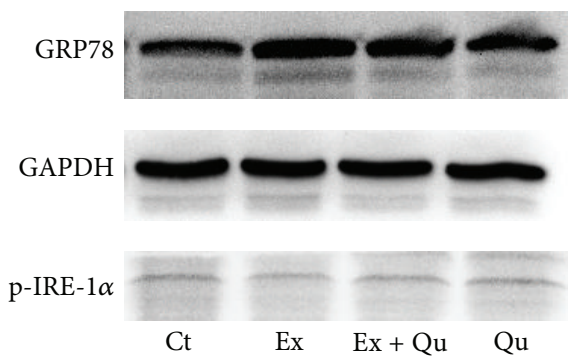

(b)

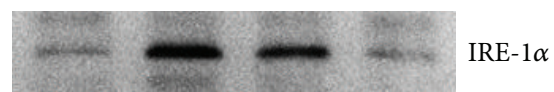

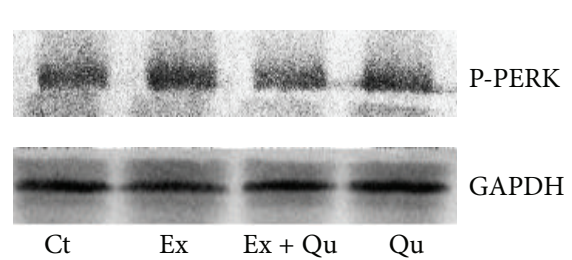

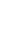

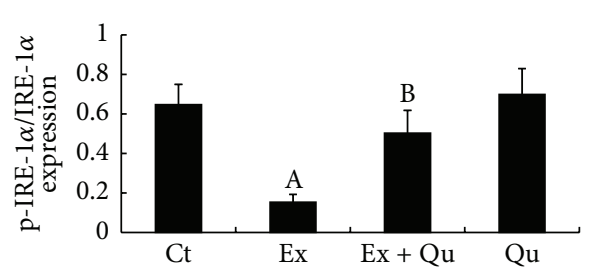

(d)

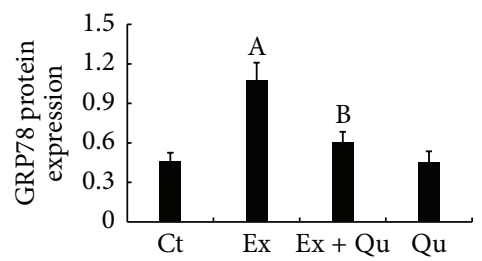

(c)

FIGURE 3: Influence of quercetin on ERS resulting from intense exercise exposure in mouse liver. The GRP78 (a, b, and c), p-IRE-1 $\alpha$ (b and $\mathrm{d})$, and p-PERK (b and e) protein expression levels were determined. The data are presented as mean $\pm \mathrm{SD}(N=3)$. Significant differences $(P<0.05)$ are indicated by the different letters. A: versus Ct; B: versus Ex. 


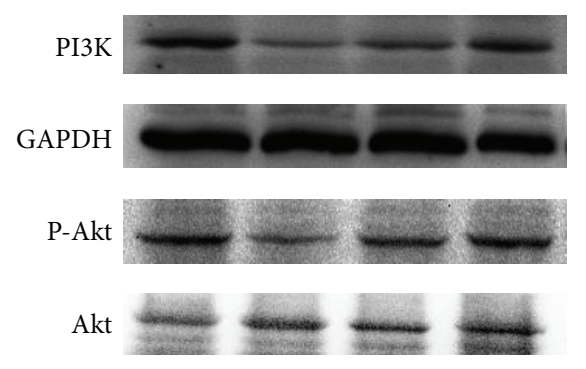

(a)

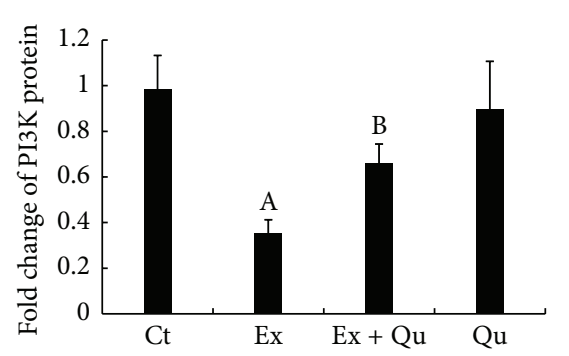

(b)

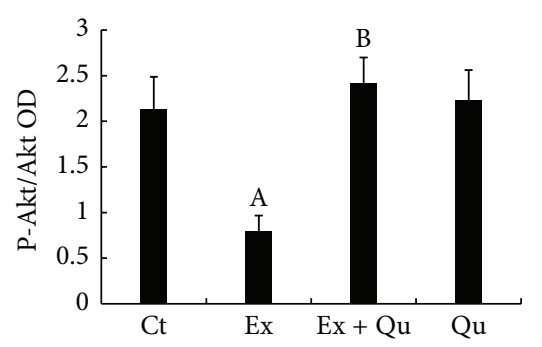

(c)

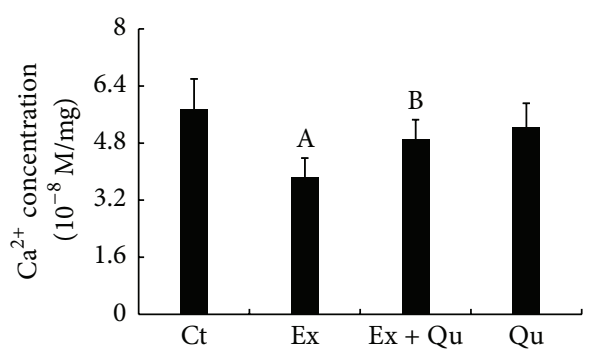

(d)

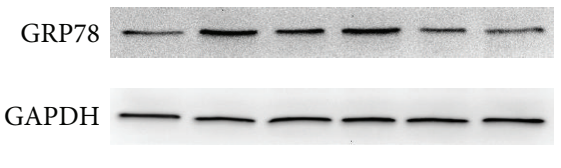

(e)

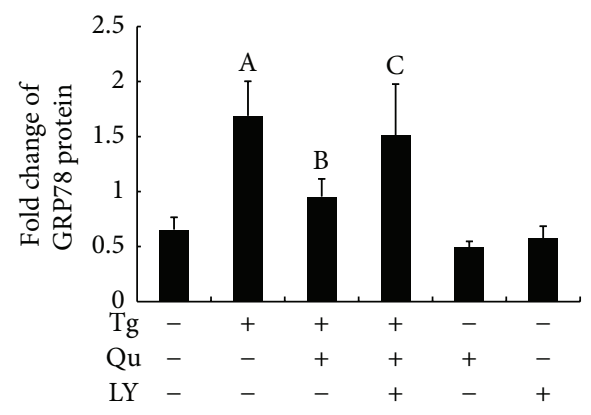

(f)

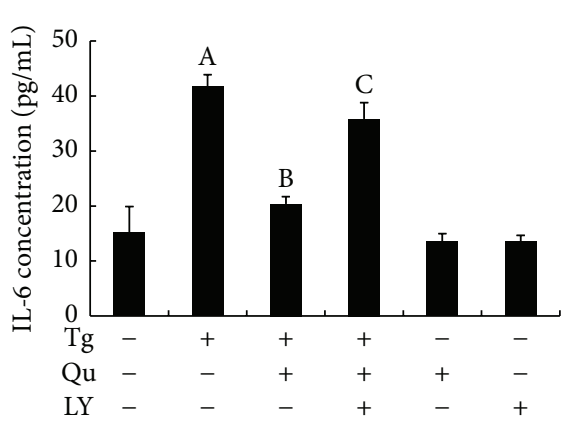

(g)

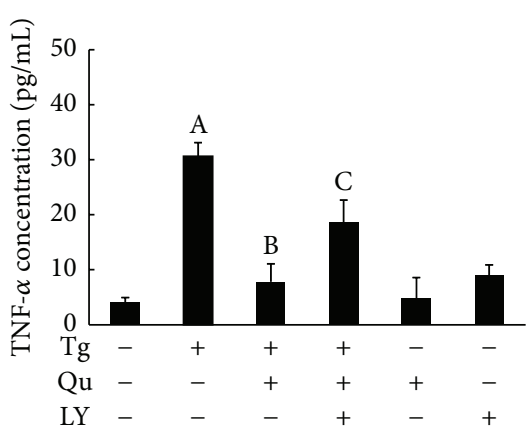

(h)

FIGURE 4: PI3K/Akt, $\mathrm{Ca}^{2+}$ dynamics, and ERS following intense exercise/Tg treatment and/or quercetin preconditioning. Hepatic PI3K (a and b) and Akt (a and c) protein levels, the $\mathrm{Ca}^{2+}$ concentration (d) in the ER, the GRP78 protein level (e and f) in mouse primary hepatocytes, and the amounts of IL-6 (g) and TNF- $\alpha(\mathrm{h})$ released from mouse primary hepatocytes into the culture medium were assessed. The data are presented as the mean $\pm \mathrm{SD}\left(N=10\right.$ for both the $\mathrm{Ca}^{2+}$ concentration determinations and ELISA, $N=3$ for others). Significant differences $(P<0.05)$ are indicated by different letters. A: versus normal control $(\mathrm{Ct})$; B: versus Tg; C: versus $\mathrm{Qu}+\mathrm{Tg}$ : quercetin $(100 \mu \mathrm{M})$ plus Tg; $\mathrm{Qu}+$ Tg + LY: quercetin + Tg + LY294002 (30 nM).

quercetin on ERS and inflammatory injury were partially abolished by preincubation of mouse primary hepatocytes with a PI3K inhibitor, LY294002. Taken together, these results indicate that $\mathrm{Ca}^{2+}$ mediates the preventive effects of quercetin on ERS via the PI3K/Akt pathway and alleviates inflammation.

\subsection{Potential Signaling Pathway Associated with ERS-Media-} ted Inflammatory Damage in Quercetin-Mediated Prophylaxis against Intense Exercise-Stimulated Hepatotoxicity. To investigate the signal transduction mechanisms, we examined the effects of the well-studied cross-talks among three molecules, that is, NF- $\kappa \mathrm{B}$, JNK, and ATF6, on the quercetin-induced protection against ERS-triggered inflammatory damage. The core components of the classical activation pathway of NF$\kappa \mathrm{B}$, p65, and p50 were determined by Western blot analyses of nuclear and cytoplasmic extracts. As illustrated in Figures 5(a) and 5(b), the nuclear translocation of p65 and p50 was evidently promoted by intense exercise stimulation compared with that in the normal control mice. Similarly, the NF- $\kappa \mathrm{B}, \mathrm{ATF} 6$, and $\mathrm{p}$-JNK levels were also significantly increased by intense exercise exposure (Figures 5(c) and 5(d)). Compared with the levels of JNK and ATF6, that of NF- $\kappa$ B exhibited a greater increase as a result of continuous intense exercise. Impressively, we observed marked decreases in the NF- $\kappa$ B, ATF6, and p-JNK levels in the quercetintreated mice following intense exercise, accompanying the reduction in inflammatory cytokines. These results imply that the quercetin-induced alleviation of ERS and inflammatory injury may be correlated with intense exercise-evoked signal transduction, especially NF- $\kappa \mathrm{B}$ nuclear translocation.

To further characterize the roles of the above signal transduction mechanisms, mouse primary hepatocytes were preincubated with PDTC (an NF- $\kappa$ B inhibitor, $300 \mu \mathrm{M}$ ), 


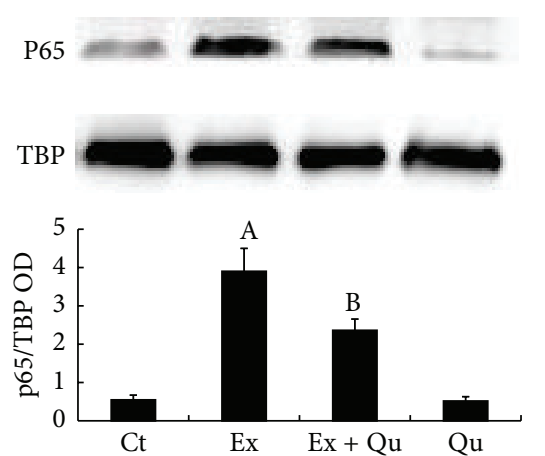

(a)

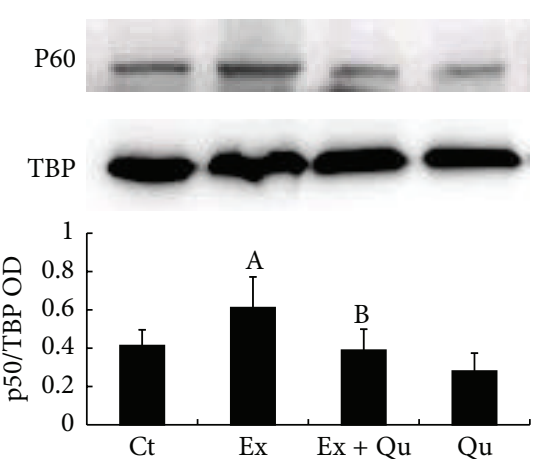

(b)

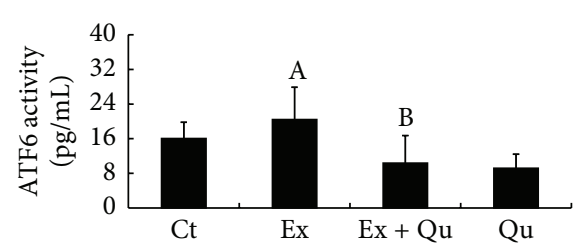

(c)
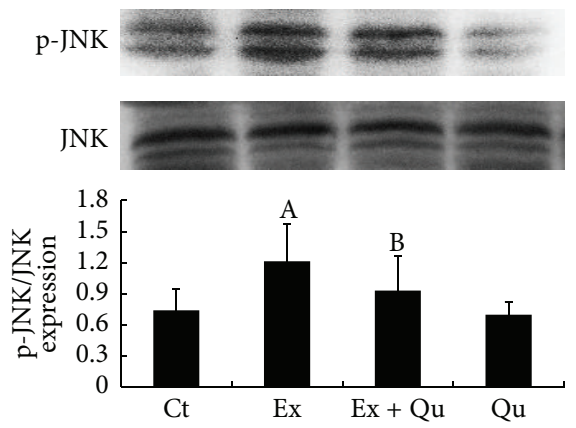

(d)

FIGURE 5: Protein expression of NF- $\kappa \mathrm{B}$ (p65 and p50 subunits) and JNK and activity level of ATF6 in mouse liver following intense exercise exposure with or without quercetin treatment. p65 (a) and p50 (b) subunit protein expression, ATF6 activity (c), and JNK protein expression (d) in nuclear fractions were determined. Three independent mouse liver isolations were performed for the protein measurements, in addition to the eight for ELISA. Significant differences $(P<0.05)$ are indicated by the different letters. A: versus Ct; B: versus Ex.

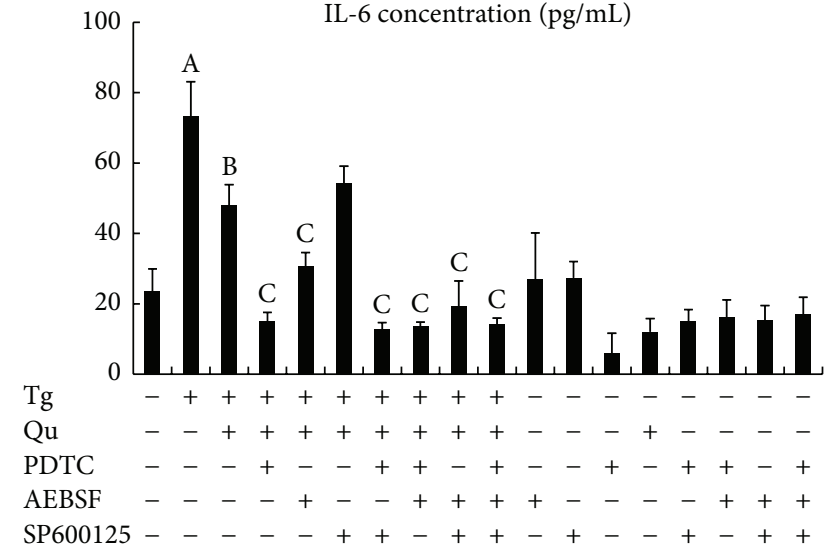

(a)

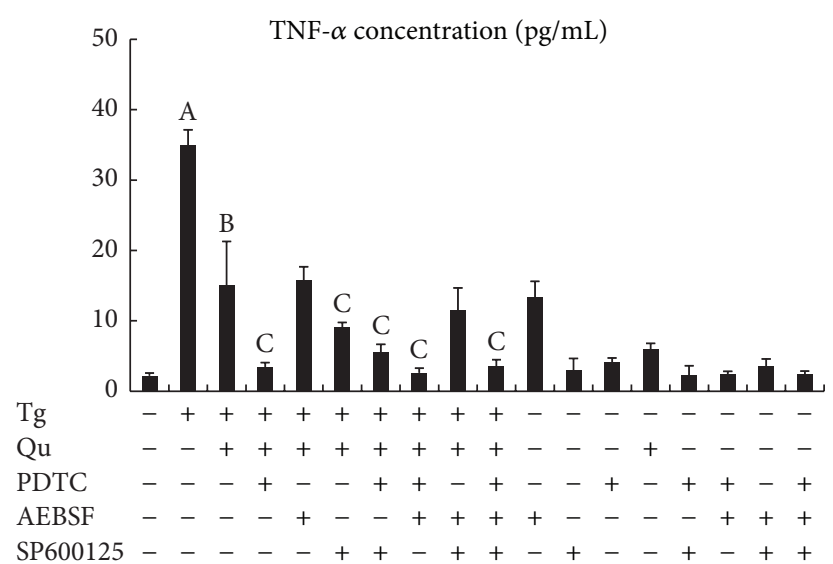

(b)

FIGURE 6: Effects of quercetin and Tg on the release of IL-6 (a) and TNF- $\alpha$ (b) from mouse primary hepatocytes and the corresponding signaling pathways involved in ERS-mediated inflammation. The data are expressed as the mean \pm SD from at least six independent hepatocyte isolations performed in triplicate. Significant differences $(P<0.05)$ are indicated by the different letters. A: versus normal control $(\mathrm{Ct}) ; \mathrm{B}$ : versus Tg; C: versus $\mathrm{Qu}+\mathrm{Tg}$.

SP600125 (a JNK inhibitor, $20 \mu \mathrm{M}$ ), and AEBSF (an ATF6 inhibitor, $300 \mu \mathrm{M})$ for 2 hours before $\mathrm{Tg}$ treatment and quercetin intervention. As shown in Figure 6, PDTC and SP600125 separately or synergistically inhibited the release of TNF- $\alpha$ from hepatocytes coincubated with Tg and quercetin.
However, SP600125 combined with Tg and quercetin treatment had no dramatic effects on IL-6 release in hepatocytes. Furthermore, PDTC exposure resulted in a significant decrease in IL- 6 to a level similar to that in untreated hepatocytes. Remarkably, PDTC had a stronger inhibitory effect on 
quercetin-mediated protection against ERS-induced inflammatory damage than SP600125. Furthermore, cotreatment with PDTC and AEBSF led to reduced levels of inflammatory cytokines (TNF- $\alpha$ and IL-6) in Tg- and quercetin-incubated hepatocytes but higher levels than those in normal controls. Treatment with AEBSF alone had no significant influence on the TNF- $\alpha$ level in Tg-treated hepatocytes. Notably, dramatically reduced inflammatory cytokine levels were observed following treatment of the Tg- and quercetin-exposed hepatocytes with the three inhibitors together. Treatment with each inhibitor alone had no effect on intact mouse hepatocytes. Furthermore, the inhibitors did not exhibit any cytotoxicity.

Collectively, these findings indicate that the PI3K/NF- $\kappa \mathrm{B}$ pathway plays a crucial role in the protective effects of quercetin against intense exercise/ERS-triggered inflammatory damage in hepatocytes.

\section{Discussion}

In the present study, intense exercise significantly induced liver damage, resulting in aminotransferase release, oxidative stress, and ultrastructural abnormalities, in agreement with the results of previous studies [4, 6, 7, 31]. Accumulating evidence has revealed that inflammation, a well-described series of events initiated by tissue injury, plays major deleterious roles in several conditions (e.g., sepsis and rheumatoid arthritis). Furthermore, inflammation is especially associated with the pathogenesis of liver diseases [32]. The observed increases in the IL- 6 and TNF- $\alpha$ levels, in accordance with other in vivo observations $[2,4,33]$, further support the crucial role of inflammatory stress in intense exercise. In current study, quercetin treatment $(100 \mathrm{mg} / \mathrm{kg} \cdot \mathrm{bw}$ for 5 weeks) effectively attenuated inflammatory stress during intense exercise stimulation and $\mathrm{Tg}$ exposure both in vivo and in vitro. Most animal studies support the protection against exercise-induced oxidative stress and inflammation. Lin et al. [20] have found that quercetin-3-O-gentiobiose possessed its anti-inflammatory activity via reducing the levels of TNF- $\alpha$ and IL-6 in exhaustive swimming rats in a dose-independent manner. Our earlier research has revealed that quercetin protects the mouse liver from inflammatory stress resulting from toxic insults [25]. Similarly, quercetin has been reported to possess potential hepatoprotective effects against highfat diets [34] and nickel-induced conditions by counteracting inflammatory damage [35], whereas, in another study from HFD-fed mice, dietary quercetin dehydrate at dose of $10 \mathrm{mg} / \mathrm{kg} \cdot \mathrm{bw} / \mathrm{day}$ for 8 weeks did not ameliorate strenuous exercise-induced inflammation in skeletal muscle [36]. We supposed that animal models, supplement form and quercetin dosage partly contributed to the inconsistency. Unfortunately, only a few human trials observed quercetininduced changes in inflammation or plasma antioxidant status. Phillips et al. [37] have found that a mix of antioxidants containing quercetin (tocopherols, docosahexaenoate, and hesperetin) attenuates IL-6 release after eccentric exercise in untrained males, demonstrating the cooperative effects of quercetin and other antioxidants on inflammation, whereas Konrad et al. [38] have concluded that acute supplementation of $1000 \mathrm{mg}$ quercetin plus $400 \mathrm{mg}$ isoquercetin before heavy exertion among endurance athletes did not prevent postexercise inflammation, although it caused a strong increase in plasma quercetin levels. O'Fallon et al. [34] have shown that quercetin aglycone supplementation $(1,000 \mathrm{mg} / \mathrm{d}$, for $7 \mathrm{~d}$ before and $5 \mathrm{~d}$ after exercise) had no effect on markers of eccentric exercise-induced muscle damage or inflammation among healthy subjects, paralleling with no change in plasma IL-6 levels before and after exercise with/without quercetin supplementation. Notably, quercetin could improve exercise performance. Recently, Askari et al. [39] demonstrated that $500 \mathrm{mg}$ quercetin plus $250 \mathrm{mg}$ vitamin C daily for 8 weeks revealed positive effects on endurance performance among male students with athletic history. The negative effects of quercetin obtained from above human studies during exercise might be due to diverse exercise manner and durations, the dose and composition of dietary supplement, and subject populations, for example, healthy and untrained, or highly trained athletes who are known to possess increased antioxidant defenses. These incompatible findings from animal and human models have still to be fully elucidated. Notably, the metabolic transformation and accumulation of quercetin between animal and human could also partly explain its bioavailability variations. In human, quercetin is metabolized in small intestines, colon, liver, and kidney and is conjugated to methyl and sulfate groups and glucuronic acid to generate its major conjugates. Moreover, the specific accumulation of quercetin conjugates and corresponding bioactivity in liver and/or muscle is still unclear. In all, our present experimental data suggest that quercetin could be a promising pharmacological agent used to prevent intense exercise-related liver damage due to its naturally occurring antioxidant and antiinflammatory activities. However, further studies in human trials are still needed to be conducted to reach a clear conclusion.

In addition to its ability to decrease the production of proinflammatory cytokines, the regulation of the endogenous anti-inflammatory system by quercetin has attracted attention. Among several potential mechanisms, ERS is considered to be an initial determining factor for stress-associated pathophysiologic disorders. ERS has been well-demonstrated to play an extremely important role in the pathogenesis of inflammation-induced liver injury [16, 28]. Generally, GRP78 binds to transducers (IRE- $1 \alpha$ and PERK), which are maintained in inactive states under physiological conditions. However, our results showed that intense exercise-exposed mice exhibited marked ERS manifested by increased GRP78 expression and the subsequent activation of PERK and IRE$1 \alpha$. The dysregulation $\mathrm{Ca}^{2+}$ dynamics via PI3K/Akt has been suggested to greatly contribute to the sequestration of GRP78 away from ERS transducers. This activity accordingly evokes ERS in apoptosis-induced tumor cells [29], reoxygenated hypoxic neonatal rat cardiomyocytes [30], and intestinal epithelial cells incubated with an ERS inducer [40]. Additionally, several studies have demonstrated that the activation of PI3K promotes hepatoprotective effects from posthepatectomy, ischemic preconditioning, or cyclic AMP [41-43]. These data, to some extent, suggest that PI3K suppression may contribute to the ERS-induced inflammatory damage of 
the mouse liver following intense exercise in association with a $\mathrm{Ca}^{2+}$ disturbance.

Plant-derived flavonoids may act as nonstressful and noncytotoxic PI3K inducers and accordingly minimize cellular stress. Quercetin accumulates in liver tissue following metabolism and accounts for $60 \%-75 \%$ of dietary flavonoids [44]. Importantly, it has been reported to protect hepatocytes against oxidative stress via PI3K activation [45]. In the current study, quercetin induced PI3K activation and Akt phosphorylation and maintained a $\mathrm{Ca}^{2+}$ level in the ER, thereby reducing intense exercise-derived ERS. Furthermore, the specific PI3K inhibitor LY294002 abolished the protective effects of quercetin on GRP78 and inflammatory cytokines in Tg-treated hepatocytes. The inhibitory effect of LY294002 is well-known to be based on its ability to block the ER $\mathrm{Ca}^{2+}$ ATPase SERCA, which is responsible for transporting $\mathrm{Ca}^{2+}$ from the cytosol to the ER [46]. PI3K activation has been suggested to lead to the recovery of SERCA and consequently to $\mathrm{Ca}^{2+}$ homeostasis [47]. Accordingly, these findings imply that regulation of the $\mathrm{PI} 3 \mathrm{~K} / \mathrm{Akt}$ signaling pathway contributes, to some extent, to the protective effects of quercetin against ERS. Conversely, opposite effects of quercetin on PI3K in LS180 cells and Caco-2 cells have been reported by another group, indicating a dual role of quercetin in PI3K activation [40]. Differing doses and durations of exposure to ERS inducers likely contributed to these reported inconsistencies.

The present study also explored the potential mechanisms of ERS-mediated inflammation in quercetin hepatoprotection. A number of studies $[13,35]$ have demonstrated that NF$\kappa \mathrm{B}, \mathrm{JNK}$, and ATF6 are critical for transducing ERS signals to initiate inflammatory responses. The activity of NF- $\kappa \mathrm{B}$, as demonstrated by $\mathrm{p} 65$ and $\mathrm{p} 50$ nuclear translocation, exhibited a greater increase compared with the activities of JNK and ATF6. Furthermore, the release of IL- 6 from hepatocytes coincubated with quercetin and $\mathrm{Tg}$ was significantly reduced by AEBSF. Similarly, SP600125 decreased the release of TNF- $\alpha$ in quercetin- and Tg-treated hepatocytes. AEBSF had no significant effect on TNF- $\alpha$, and SP600125 did not further block IL-6 release from hepatocytes following quercetin and $\mathrm{Tg}$ treatment. Both inflammatory cytokines were significantly blocked by the NF- $\kappa \mathrm{B}$ inhibitors, applied singly or in combination, indicating that NF- $\kappa$ B is primarily responsible for quercetin-mediated hepatoprotection against ERS-induced inflammation. The involvement of NF- $\kappa \mathrm{B}$ in ERS-activated inflammation has also been established by other studies of the effects of 4-phenylbutyrate on lipopolysaccharide-induced lung damage [48], cardiomyocyte hypoxia/reoxygenation [49], and IL- $\beta$ production [50]. Further, quercetin has been found to inhibit NF- $\kappa$ B-mediated HepG2 cell damage [51]. Such protective effects, which occur via $\mathrm{NF}-\kappa \mathrm{B}$ sequestration, have also been observed for other flavonoids (e.g., morin and carnosol) $[52,53]$. These in vivo and in vitro data show that quercetin may suppress the expression of IL- 6 and TNF- $\alpha$ by blocking the recruitment of NF- $\kappa \mathrm{B}$ to respective gene promoters upon ERS exposure.

\section{Conclusions}

The results of this study demonstrate the beneficial effects of quercetin on liver damage following exhaustive exercise. Quercetin supplementation may be an effective strategy to offset increases in oxidative stress, inflammation, and ERS, which have been linked with stressful exercise. These results may lead to the development of nutritional regimens that decrease the risk of liver injury, which can be an issue for military personnel and athletes engaged in cycling, triathlons, cross-country skiing, rowing, and distance running. Furthermore, our findings highlight $\mathrm{PI} 3 \mathrm{~K}$ and $\mathrm{NF}-\kappa \mathrm{B}$ as potential candidate targets for the prevention of tissue damage induced by intense exercise. Despite these findings, the molecular mechanisms and interactions of ERS and inflammatory damage remain unknown.

\section{Competing Interests}

The authors declare that they have no competing interests.

\section{Acknowledgments}

This work was supported by the National Natural Science Foundation of China (nos. 81472979 and 81402673).

\section{References}

[1] D. G. Jakovljevic, K. Hallsworth, P. Zalewski et al., "Resistance exercise improves autonomic regulation at rest and haemodynamic response to exercise in non-alcoholic fatty liver disease," Clinical Science, vol. 125, no. 3, pp. 143-149, 2013.

[2] M. A. Febbraio, "Exercise and inflammation," Journal of Applied Physiology, vol. 103, no. 1, pp. 376-377, 2007.

[3] K. Brooks and J. Carter, "Overtraining, exercise, and adrenal insufficiency," Journal of Novel Physiotherapies, vol. 3, article 125, 2013.

[4] E. Fojt, L.-G. Ekelund, and E. Hultman, "Enzyme activities in hepatic venous blood under strenuous physical exercise," Pflügers Archiv European Journal of Physiology, vol. 361, no. 3, pp. 287-296, 1976.

[5] F. D. Lima, D. N. Stamm, I. D. Della-Pace et al., "Swimming training induces liver mitochondrial adaptations to oxidative stress in rats submitted to repeated exhaustive swimming bouts," PLoS ONE, vol. 8, no. 2, Article ID e55668, 2013.

[6] C.-C. Huang, W.-T. Lin, F.-L. Hsu, P.-W. Tsai, and C.-C. Hou, "Metabolomics investigation of exercise-modulated changes in metabolism in rat liver after exhaustive and endurance exercises," European Journal of Applied Physiology, vol. 108, no. 3, pp. 557-566, 2010.

[7] P. Praphatsorn, D. Thong-Ngama, O. Kulaputana, and N. Klaikeaw, "Effects of intense exercise on biochemical and histological changes in rat liver and pancreas," Asian Biomedicine, vol. 4, no. 4, pp. 619-625, 2010.

[8] A. La Gerche and H. Heidbuchel, "Can intensive exercise harm the heart? You can get too much of a good thing," Circulation, vol. 130, no. 12, pp. 992-1002, 2014.

[9] H.-T. Yang, L.-J. Luo, W.-J. Chen et al., "IL-15 expression increased in response to treadmill running and inhibited endoplasmic reticulum stress in skeletal muscle in rats," Endocrine, vol. 48, no. 1, pp. 152-163, 2015. 
[10] E.-B. Kang, I.-S. Kwon, J.-H. Koo et al., “Treadmill exercise represses neuronal cell death and inflammation during $\mathrm{A} \beta$ induced ER stress by regulating unfolded protein response in aged presenilin 2 mutant mice," Apoptosis, vol. 18, no. 11, pp. 1332-1347, 2013.

[11] L. Deldicque, P. D. Cani, N. M. Delzenne, K. Baar, and M. Francaux, "Endurance training in mice increases the unfolded protein response induced by a high-fat diet," Journal of Physiology and Biochemistry, vol. 69, no. 2, pp. 215-225, 2013.

[12] H. J. Kim, C. Jamart, L. Deldicque et al., "Endoplasmic reticulum stress markers and ubiquitin-proteasome pathway activity in response to a 200-km run," Medicine and Science in Sports and Exercise, vol. 43, no. 1, pp. 18-25, 2011.

[13] K. Zhang and R. J. Kaufman, "From endoplasmic-reticulum stress to the inflammatory response," Nature, vol. 454, no. 7203, pp. 455-462, 2008.

[14] Y. Michailidis, L. G. Karagounis, G. Terzis et al., "Thiol-based antioxidant supplementation alters human skeletal muscle signaling and attenuates its inflammatory response and recovery after intense eccentric exercise," The American Journal of Clinical Nutrition, vol. 98, no. 1, pp. 233-245, 2013.

[15] Y. Kim, M. Park, S. Boghossian, and D. A. York, "Three weeks voluntary running wheel exercise increases endoplasmic reticulum stress in the brain of mice," Brain Research, vol. 1317, pp. 13-23, 2010.

[16] R. He, J. Feng, Q. Xun, Q. Qin, and C. Hu, "High-intensity training induces EIB in rats through neuron transdifferentiation of adrenal medulla chromaffin cells," American Journal of Physiology_Lung Cellular and Molecular Physiology, vol. 304, no. 9, pp. L602-L612, 2013.

[17] I. Erlund, "Review of the flavonoids quercetin, hesperetin, and naringenin. Dietary sources, bioactivities, bioavailability, and epidemiology," Nutrition Research, vol. 24, no. 10, pp. 851-874, 2004.

[18] J. Kressler, M. Millard-Stafford, and G. L. Warren, "Quercetin and endurance exercise capacity: a systematic review and metaanalysis," Medicine and Science in Sports and Exercise, vol. 43, no. 12, pp. 2396-2404, 2011.

[19] P. Yao, A. Nussler, L. Liu et al., "Quercetin protects human hepatocytes from ethanol-derived oxidative stress by inducing heme oxygenase-1 via the MAPK/Nrf2 pathways," Journal of Hepatology, vol. 47, no. 2, pp. 253-261, 2007.

[20] Y. Lin, H.-L. Liu, J. Fang, C.-H. Yu, Y.-K. Xiong, and K. Yuan, "Anti-fatigue and vasoprotective effects of quercetin-3O-gentiobiose on oxidative stress and vascular endothelial dysfunction induced by endurance swimming in rats," Food and Chemical Toxicology, vol. 68, pp. 290-296, 2014.

[21] C.-M. Liu, G. H. Zheng, Q. L. Ming, J. M. Sun, and C. Cheng, "Protective effect of quercetin on lead-induced oxidative stress and endoplasmic reticulum stress in rat liver via the IRE1/JNK and PI3K/Akt pathway," Free Radical Research, vol. 47, no. 3, pp. 192-201, 2013.

[22] A. B. Granado-Serrano, M. Á. Martín, L. Bravo, L. Goya, and S. Ramos, "Quercetin attenuates TNF-induced inflammation in hepatic cells by inhibiting the NF- $\kappa \mathrm{B}$ pathway," Nutrition and Cancer, vol. 64, no. 4, pp. 588-598, 2012.

[23] E. Marcolin, B. San-Miguel, D. Vallejo et al., "Quercetin treatment ameliorates inflammation and fibrosis in mice with nonalcoholic steatohepatitis," Journal of Nutrition, vol. 142, no. 10, pp. 1821-1828, 2012.

[24] S. Marra, M. Burnett, and L. Hoffman-Goetz, "Intravenous catecholamine administration affects mouse intestinal lymphocyte number and apoptosis," Journal of Neuroimmunology, vol. 158, no. 1-2, pp. 76-85, 2005.

[25] Y. Li, C. Gao, Y. Shi et al., "Carbon monoxide alleviates ethanolinduced oxidative damage and inflammatory stress through activating p38 MAPK pathway," Toxicology and Applied Pharmacology, vol. 273, no. 1, pp. 53-58, 2013.

[26] Y. H. Tang, C. Gao, Y. R. Shi et al., "Quercetin attenuates ethanol-derived microsomal oxidative stress: implication of haem oxygenase-1 induction," Food Chemistry, vol. 132, no. 4, pp. 1769-1774, 2012.

[27] W. Cao, Q. F. Collins, T. C. Becker et al., "p38 Mitogenactivated protein kinase plays a stimulatory role in hepatic gluconeogenesis," The Journal of Biological Chemistry, vol. 280, no. 52, pp. 42731-42737, 2005.

[28] L. Dara, C. Ji, and N. Kaplowitz, "The contribution of endoplasmic reticulum stress to liver diseases," Hepatology, vol. 53, no. 5, pp. 1752-1763, 2011.

[29] M.-L. Lin, S.-S. Chen, R.-Y. Huang et al., "Suppression of PI3K/Akt signaling by synthetic bichalcone analog TSWU-CD4 induces ER stress- and Bax/Bak-mediated apoptosis of cancer cells," Apoptosis, vol. 19, no. 11, pp. 1637-1653, 2014.

[30] J. Guo, Y. Bian, R. Bai, H. Li, M. Fu, and C. Xiao, "Globular adiponectin attenuates myocardial ischemia/reperfusion injury by upregulating endoplasmic reticulum $\mathrm{Ca}^{2+}$-Atpase activity and inhibiting endoplasmic reticulum stress," Journal of Cardiovascular Pharmacology, vol. 62, no. 2, pp. 143-153, 2013.

[31] E. Kerasioti, A. Kiskini, A. Veskoukis et al., "Effect of a special carbohydrate-protein cake on oxidative stress markers after exhaustive cycling in humans," Food and Chemical Toxicology, vol. 50, no. 8, pp. 2805-2810, 2012.

[32] H. Tilg and A. R. Moschen, "Evolution of inflammation in nonalcoholic fatty liver disease: the multiple parallel hits hypothesis," Hepatology, vol. 52, no. 5, pp. 1836-1846, 2010.

[33] E. Kerasioti, D. Stagos, A. Jamurtas et al., "Anti-inflammatory effects of a special carbohydrate-whey protein cake after exhaustive cycling in humans," Food and Chemical Toxicology, vol. 61, pp. 42-46, 2013.

[34] K. S. O’Fallon, D. Kaushik, B. Michniak-Kohn, C. P. Dunne, E. J. Zambraski, and P. M. Clarkson, "Effects of quercetin supplementation on markers of muscle damage and inflammation after eccentric exercise," International Journal of Sport Nutrition and Exercise Metabolism, vol. 22, no. 6, pp. 430-437, 2012.

[35] C.-M. Liu, J.-Q. Ma, W.-R. Xie et al., "Quercetin protects mouse liver against nickel-induced DNA methylation and inflammation associated with the Nrf2/HO-1 and p38/STAT1/NF- $\kappa \mathrm{B}$ pathway," Food and Chemical Toxicology, vol. 82, pp. 19-26, 2015.

[36] S. M. Kwon, H. G. Park, J. K. Jun, and W. L. Lee, "Exercise, but not quercetin, ameliorates inflammation, mitochondrial biogenesis, and lipid metabolism in skeletal muscle after strenuous exercise by high-fat diet mice," Journal of Exercise Nutrition and Biochemistry, vol. 18, no. 1, pp. 51-60, 2014.

[37] T. Phillips, A. C. Childs, D. M. Dreon, S. Phinney, and C. Leeuwenburgh, "A dietary supplement attenuates IL-6 and CRP after eccentric exercise in untrained males," Medicine and Science in Sports and Exercise, vol. 35, no. 12, pp. 2032-2037, 2003.

[38] M. Konrad, D. C. Nieman, D. A. Henson, K. M. Kennerly, F. Jin, and S. J. Wallner-Liebmann, "The acute effect of ingesting a quercetin-based supplement on exercise-induced inflammation and immune changes in runners," International Journal of Sport Nutrition and Exercise Metabolism, vol. 21, no. 4, pp. 338-346, 2011. 
[39] G. Askari, R. Ghiasvand, Z. Paknahad et al., "The effects of quercetin supplementation on body composition, exercise performance and muscle damage indices in athletes," International Journal of Preventive Medicine, vol. 4, no. 1, pp. 21-26, 2013.

[40] Y. Natsume, S. Ito, H. Satsu, and M. Shimizu, "Protective effect of quercetin on ER stress caused by calcium dynamics dysregulation in intestinal epithelial cells," Toxicology, vol. 258, no. 2-3, pp. 164-175, 2009.

[41] M. Cescon, R. Carini, G. Grazi et al., "Variable activation of phosphoinositide 3-kinase influences the response of liver grafts to ischemic preconditioning," Journal of Hepatology, vol. 50, no. 5, pp. 937-947, 2009.

[42] Y. Nechemia-Arbely, A. Shriki, U. Denz et al., "Early hepatocyte DNA synthetic response posthepatectomy is modulated by IL-6 trans-signaling and PI3K/AKT activation," Journal of Hepatology, vol. 54, no. 5, pp. 922-929, 2011.

[43] B. C. Zhang, S. Li, and B. G. Harbrecht, "Akt-mediated signaling is induced by cytokines and cyclic adenosine monophosphate and suppresses hepatocyte inducible nitric oxide synthase expression independent of MAPK P44/42," Biochimica et Biophysica Acta (BBA)-Molecular Cell Research, vol. 1813, no. 1, pp. 73-79, 2011.

[44] L. Sampson, E. Rimm, P. C. H. Hollman, J. H. M. de Vries, and M. B. Katan, "Flavonol and flavone intakes in US health professionals," Journal of the American Dietetic Association, vol. 102, no. 10, pp. 1414-1420, 2002.

[45] C.-J. Weng, M.-J. Chen, C.-T. Yeh, and G.-C. Yen, "Hepatoprotection of quercetin against oxidative stress by induction of metallothionein expression through activating MAPK and PI3K pathways and enhancing Nrf2 DNA-binding activity," New Biotechnology, vol. 28, no. 6, pp. 767-777, 2011.

[46] Y. Imai, H. Yamagishi, Y. Ono, and Y. Ueda, "Versatile inhibitory effects of the flavonoid-derived PI3K/Akt inhibitor, LY294002, on ATP-binding cassette transporters that characterize stem cells," Clinical and Translational Medicine, vol. 1, article 24, 2012.

[47] J. DeSantiago, D. J. Bare, I. Semenov et al., "Excitation-contraction coupling in ventricular myocytes is enhanced by paracrine signaling from mesenchymal stem cells," Journal of Molecular and Cellular Cardiology, vol. 52, no. 6, pp. 1249-1256, 2012.

[48] H. J. Kim, J. S. Jeong, S. R. Kim, S. Y. Park, H. J. Chae, and Y. C. Lee, "Inhibition of endoplasmic reticulum stress alleviates lipopolysaccharide- induced lung inflammation through modulation of NF- $\kappa$ B/HIF- $1 \alpha$ signaling pathway," Scientific Reports, vol. 3, article 1142, 2013.

[49] Q. Wu, Q. Wang, Z. Guo, Y. Shang, L. Zhang, and S. Gong, "Nuclear factor- $\kappa \mathrm{B}$ as a link between endoplasmic reticulum stress and inflammation during cardiomyocyte hypoxia/reoxygenation," Cell Biology International, vol. 38, no. 7, pp. 881-887, 2014.

[50] S. Kim, Y. Joe, S. O. Jeong et al., "Endoplasmic reticulum stress is sufficient for the induction of IL- $1 \beta$ production via activation of the NF- $\kappa \mathrm{B}$ and inflammasome pathways," Innate Immunity, vol. 20, no. 8, pp. 799-815, 2014.

[51] C. A. Musonda and J. K. Chipman, "Quercetin inhibits hydrogen peroxide $\left(\mathrm{H}_{2} \mathrm{O}_{2}\right)$-induced NF- $\kappa \mathrm{B}$ DNA binding activity and DNA damage in HepG2 cells," Carcinogenesis, vol. 19, no. 9, pp. 1583-1589, 1998.

[52] J. M. Kim, E. K. Lee, G. Park et al., "Morin modulates the oxidative stress-induced NF- $\kappa \mathrm{B}$ pathway through its anti-oxidant activity," Free Radical Research, vol. 44, no. 4, pp. 454-461, 2010.

[53] A.-H. Lo, Y.-C. Liang, S.-Y. Lin-Shiau, C.-T. Ho, and J.-K. Lin, "Carnosol, an antioxidant in rosemary, suppresses inducible nitric oxide synthase through down-regulating nuclear factor$\kappa \mathrm{B}$ in mouse macrophages," Carcinogenesis, vol. 23 , no. 6 , pp. 983-991, 2002. 


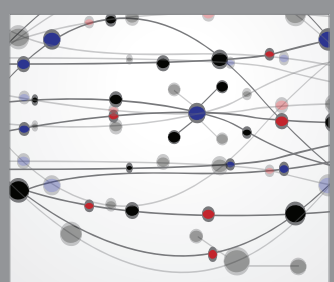

The Scientific World Journal
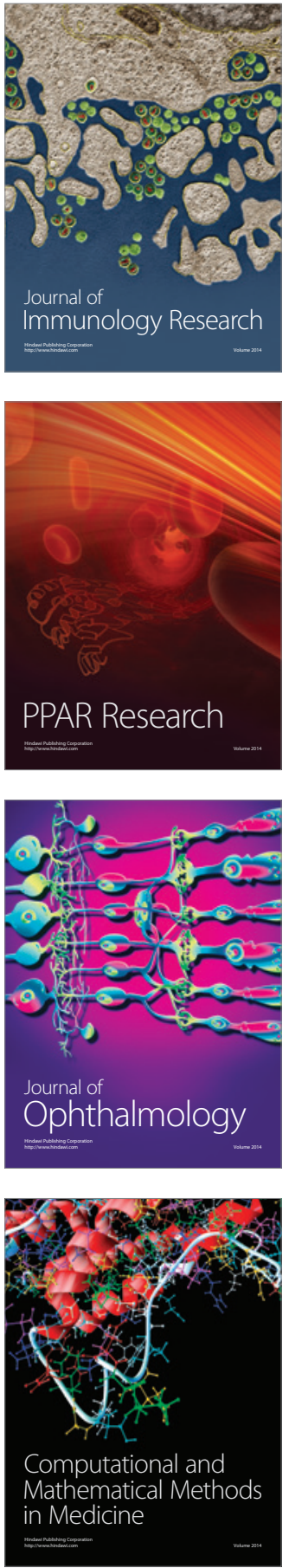

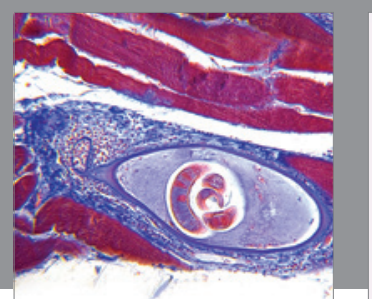

Gastroenterology Research and Practice

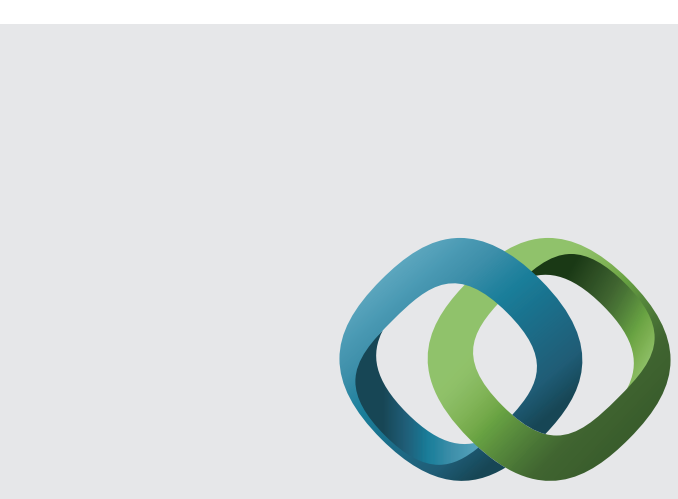

\section{Hindawi}

Submit your manuscripts at

http://www.hindawi.com
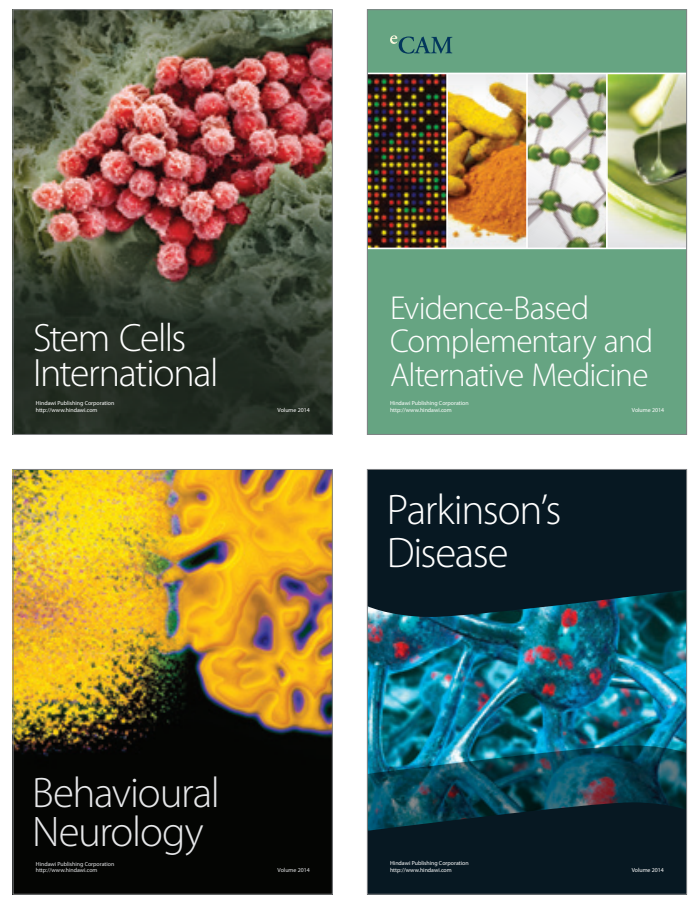
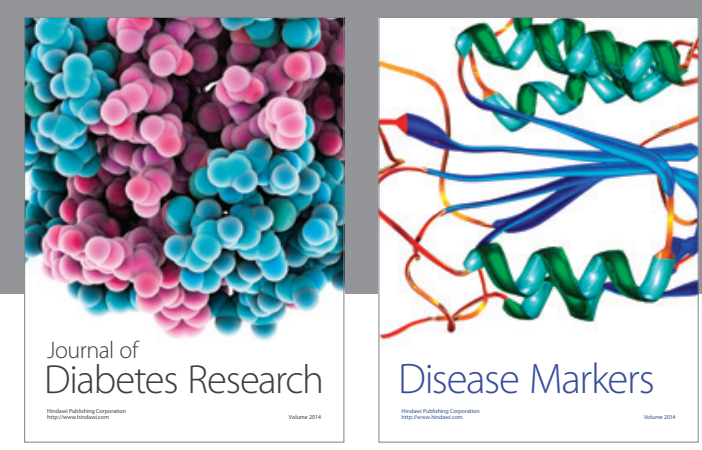

Disease Markers
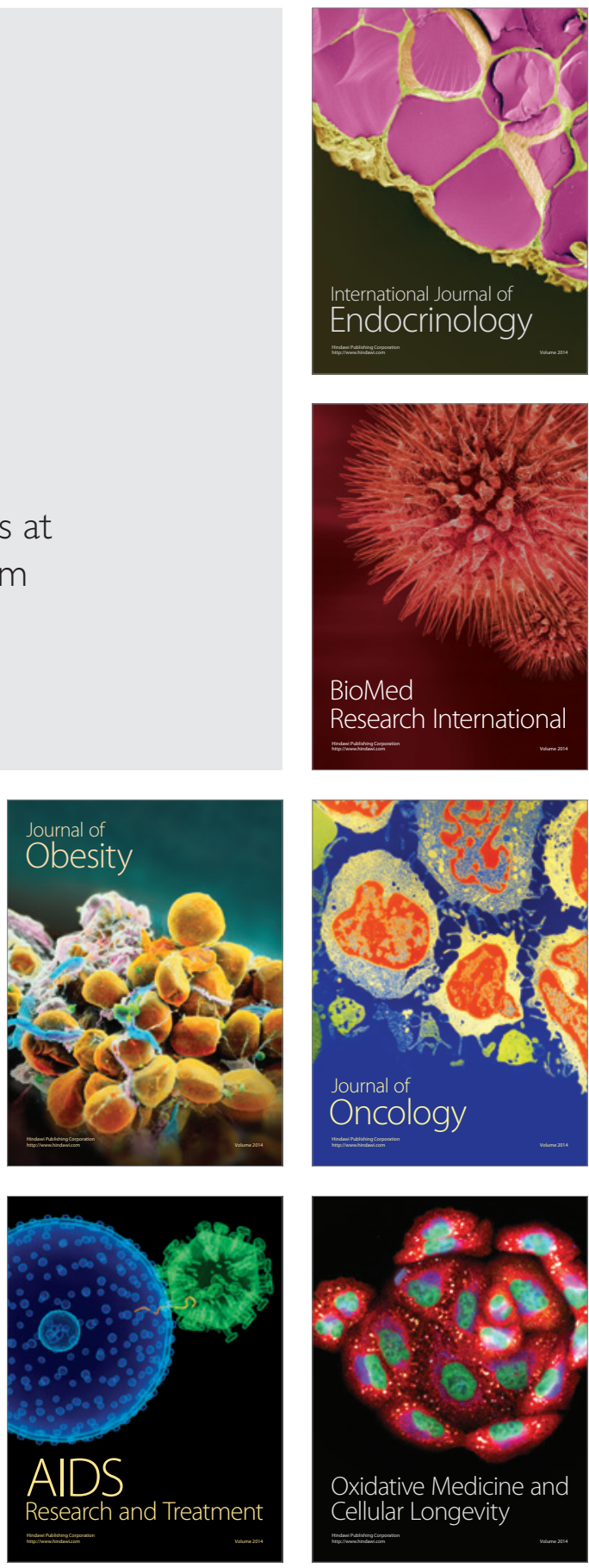